\title{
A genome-wide association study explores the genetic determinism of host resistance to Salmonella pullorum infection in chickens
}

Xinghua Li ${ }^{1}$, Changsheng Nie' ${ }^{1}$ Yuchen Liu' ${ }^{1}$ Yu Chen ${ }^{2}$, Xueze LV², Liang Wang ${ }^{2}$, Jianwei Zhang ${ }^{2}$, Kaiyang Li ${ }^{2}$, Yaxiong Jia ${ }^{3}$, Liping Ban ${ }^{4^{*}}$, Zhonghua Ning ${ }^{1 *}$ and Lujiang Qu ${ }^{1^{*}}$ (D

\begin{abstract}
Background: Salmonella infection is a serious concern in poultry farming because of its impact on both economic loss and human health. Chicks aged 20 days or less are extremely vulnerable to Salmonella pullorum (SP), which causes high mortality. Furthermore, an outbreak of SP infection can result in a considerable number of carriers that become potential transmitters, thus, threatening fellow chickens and offspring. In this study, we conducted a genome-wide association study (GWAS) to detect potential genomic loci and candidate genes associated with two disease-related traits: death and carrier state.
\end{abstract}

Methods: In total, 818 birds were phenotyped for death and carrier state traits through a SP challenge experiment, and genotyped by using a $600 \mathrm{~K}$ high-density single nucleotide polymorphism (SNP) array. A GWAS using a singlemarker linear mixed model was performed with the GEMMA software. RNA-sequencing on spleen samples was carried out for further identification of candidate genes.

Results: We detected a region that was located between 33.48 and $34.03 \mathrm{Mb}$ on chicken chromosome 4 and was significantly associated with death, with the most significant SNP (rs314483802) accounting for $11.73 \%$ of the phenotypic variation. Two candidate genes, FBXW7 and $\angle R B A$, were identified as the most promising genes involved in resistance to SP. The expression levels of FBXW7 and $\angle R B A$ were significantly downregulated after SP infection, which suggests that they may have a role in controlling SP infections. Two other significant loci and related genes (TRAF3 and gga-mir-489) were associated with carrier state, which indicates a different polygenic determinism compared with that of death. In addition, genomic inbreeding coefficients showed no correlation with resistance to SP within each breed in our study.

Conclusions: The results of this GWAS with a carefully organized Salmonella challenge experiment represent an important milestone in understanding the genetics of infectious disease resistance, offer a theoretical basis for breeding SP-resistant chicken lines using marker-assisted selection, and provide new information for salmonellosis research in humans and other animals.

\footnotetext{
*Correspondence: lipingban@cau.edu.cn; ningzhh@cau.edu.cn; quluj@163.com

${ }^{1}$ State Key Laboratory of Animal Nutrition, Department of Animal Genetics and Breeding, National Engineering Laboratory for Animal Breeding, College of Animal Science and Technology, China Agricultural University, Beijing, China

${ }^{4}$ Department of Grassland Science, China Agricultural University, Beijing,

China

Full list of author information is available at the end of the article
} (http://creativecommons.org/licenses/by/4.0/), which permits unrestricted use, distribution, and reproduction in any medium, provided you give appropriate credit to the original author(s) and the source, provide a link to the Creative Commons license, and indicate if changes were made. The Creative Commons Public Domain Dedication waiver (http://creativecommons.org/ publicdomain/zero/1.0/) applies to the data made available in this article, unless otherwise stated. 


\section{Background}

Salmonella infection is a serious concern in poultry farming. On the one hand, systemic salmonellosis results in considerable animal mortality and reduced poultry production. On the other hand, poultry is a major global reservoir of nontyphoidal Salmonellae, which is one of the most important pathogens that cause foodborne illnesses [1]. Pullorum disease is an acute poultry infectious disease that is caused by the chicken-restricted facultative intracellular gram-negative bacterium, Salmonella pullorum (SP). This disease usually results in high mortality of chicks less than 20 days old, especially in developing countries where cleaning and disinfection procedures are usually not effective [2]. SP infection generally leads to three disease outcomes: the most susceptible birds die within about 2 to 20 days showing typical SP infection symptoms such as hepatosplenomegaly, white diarrhea and cecal cores [3]; some chicks survive by clearing the pathogen through a series of immune responses; and other chicks develop a carrier state with SP present in their splenic macrophages for a long period of time [4]. These carriers can transmit the pathogen to other chickens horizontally or to their offspring via the eggs [5].

In many developed countries, the pullorum disease has been eradicated from commercial flocks by culling infected birds. However, this method does not work well in most developing countries due to the emergence of novel bacterial strains [6], poor hygienic conditions, and limited technology; in addition, there are restrictions on the use of antibiotics in food animal production. Thus, there is need for an alternative sustainable strategy to control the disease in farm animals. In addition to novel vaccines and food additives, selective breeding of animals based on the development of chicken genomic data is becoming a promising approach to improve their resistance to infectious diseases [7].

Host genetic factors have been reported to play an important role in the resistance of animals to Salmonella infection in many studies [8-14]. Previously, we estimated the heritability of the death and carrier state traits based on an elaborately designed challenge experiment [3]. The results showed low-to-moderate heritabilities (0.09 to 0.32) in different chicken lines, which means these traits are heritable. However, the molecular mechanism that underlies the genetic resistance to SP remains largely unknown. In recent years, genome-wide association studies (GWAS) have been widely used to identify the genetic architecture of many disease traits in chickens [15-18]. However, only a few GWAS have been carried out on infectious diseases because it is difficult and expensive to obtain accurate phenotypes for large populations; furthermore, the results of an infection are affected by many factors such as bacterial dosage, maternal antibodies, and the environment [19], which are difficult to control.

To the best of our knowledge, no large-scale GWAS has been performed to identify genomic loci and candidate genes for death and carrier state, through a carefully organized SP challenge test. In this study, 818 pure-bred chicks were genotyped with a commercial $600 \mathrm{~K}$ highdensity single nucleotide polymorphism (SNP) array [20]. A GWAS using a single-marker linear mixed model and 302,927 SNPs allowed us to detect genomic regions that are associated with resistance to SP. The identified candidate genes were evaluated based on their functional annotation and expression level. The potential mechanisms of these genes in immunity to infection in chickens are discussed.

\section{Methods}

\section{Animals and phenotyping}

For this study, 842 chicks from three pure lines were available, namely 384 Rhode Island Red, 381 Dwarf Chicken, and 77 Beijing You individuals. Rhode Island Red (RIR) is an intensively selected commercial breed, Dwarf Chicken (DW) is a synthetic layer line, and Beijing You (BY) is a Chinese local chicken breed. These animals all came from our previous SP challenge experiment [3]. Briefly, SP pathogen-free and antibody-free chicks were orally inoculated with $4.8 \times 10^{7}$ colony forming units (CFU) SP strain 533 culture at 4 days of age and then raised in negative pressure isolators up to 40 days of age. All chicks had free access to sterile water and food, and the environment was managed such that it remained the same for all the birds in the experiment. Three different disease-related traits, i.e. death, clearance and carrier-state, were measured and used as SP resistance phenotypes. First, chicks that died with visible signs of SP infection symptoms (white diarrhea and splenomegaly) were classified as the most susceptible. In the case of chicks that survived the challenge, the carrier-state was analyzed by plating spleen homogenates on MacConkey agar medium under sterile conditions to determine bacterial load. The identity of the pathogen was confirmed by Sanger sequencing of the SP specific ipaJ gene [21] after PCR amplification with the following primers: sense 5'-ATTAACAGGAGGAGGCTGG-3'; antisense 5'-CCA TTCCCAAAAGCCTGCAT- $3^{\prime}$. More details on how the population was established and on the bacterial challenge process are in our previous report [3].

\section{Genotyping, quality control and imputation}

We isolated individual genomic DNA from blood or muscle samples by the classical phenol-chloroform procedure. DNA integrity was verified by agarose gel electrophoresis and purity was checked by A260/280 ratio 
using a NanoDrop 2000 spectrophotometer (Thermo Fisher Scientific $\left.{ }^{\mathrm{TM}}\right)$. In total, 842 qualified individual genomic DNA samples were genotyped using the Affymetrix $600 \mathrm{~K}$ chicken high-density array (Affymetrix, Inc. Santa Clara, CA, USA). For SNP calling and initial quality control, the raw genotyping data (CEL files) were analyzed by using the software Axiom Analysis Suite 3.1 following the Best Practices Workflow. Only the samples with a dish quality control (DQC) of 0.82 or more and a call rate higher than $95 \%$ were retained for subsequent analyses. The SNP QC metrics were set to the default values recommended by Affymetrix, except that only "PolyHighResolution" SNPs were included in our analysis. Furthermore, we excluded SNPs with unknown or repeated physical positions with an ad hoc R script. SNPs on the sex chromosomes were also discarded because the current statistical methods are not powerful enough to detect associations between phenotypes and sexrelated genotypes. After these QC steps, 818 samples and 452,291 SNPs remained. A Hardy-Weinberg equilibrium (HWE) test was conducted within each breed, and 62,450 variants that deviated from HWE $\left(\mathrm{P}<1 \times 10^{-5}\right)$ were filtered out. To increase the power of the association analysis, we removed 85,140 SNPs because of their low level of variation among subpopulations (i.e. SNPs that were monomorphic in any one of the three breeds), and 1774 SNPs with a minor allele frequency (MAF) lower than 0.05 using PLINK v1.90 [22]. Missing genotypes were imputed based on information from the remaining SNP genotypes for each subpopulation separately, according to the software Beagle Version 4 [23]. In total, 818 samples and 302,927 SNPs were included in the subsequent genome-wide association analysis.

\section{Statistical analysis}

Population structure and relatedness are major sources of confounding effects in genetic association studies [24]. The most popular method for GWAS that include related individuals is the linear mixed model (LMM) method because of its effectiveness in controlling population stratification bias and reducing the inflation from many small genetic effects (polygenic background) [25-31]. In this study, we assessed population structure by conducting a principal component analysis (PCA) implemented in the PLINK package. Considering that clusters of SNPs in high linkage disequilibrium may bias the PCA results, first we pruned the full SNP set to 23,870 independent SNPs using the-indep-pairwise 2550.2 command parameters in PLINK. Then, we used these unlinked SNPs to calculate the top three principal components (PC) that were used as covariates in the mixed model. Furthermore, a pairwise kinship matrix was built using the pruned SNPs.
A single-marker univariate linear mixed model was used for testing associations between the results of SP infection and the qualified SNPs. The disease phenotype was divided into two binary traits: death (200 deaths vs. 618 survivals) and carrier-state (161 carriers vs. 457 clearance). Both death and carrier-state were analyzed using the following model:

$$
\mathbf{y}=\mathbf{W} \boldsymbol{\alpha}+\mathbf{x} \boldsymbol{\beta}+\mathbf{u}+\boldsymbol{\varepsilon},
$$

where $\mathbf{y}$ denotes the trait values for death or carrier-state, namely either ' 0 ' or ' 1 '; $\mathbf{W}$ is a matrix of covariates (i.e. fixed effects that contain the top three PC, genotyping batch and a column of 1s) that control population structure and batch effect; $\alpha$ is a vector of corresponding coefficients that includes the intercept; $\mathbf{x}$ is a vector of SNP genotypes; $\boldsymbol{\beta}$ is the corresponding effect of SNPs; $\mathbf{u}$ is a vector of random polygenic effects with a covariance structure that follows a normal distribution $\mathbf{u} \sim \mathrm{N}\left(0, \mathbf{K V}_{\mathrm{g}}\right)$, where $\mathbf{K}$ is a genomic relationship matrix derived from independent SNPs and $V_{g}$ is the polygenic additive variance; and $\varepsilon$ is a vector of random errors. The association analysis was performed using the GEMMA v0.96 software [30]. The Wald test statistic $\mathrm{F}_{\text {wald }}=\hat{\beta}^{2} / \operatorname{Var}(\hat{\beta})$ was used to test the null hypothesis $\beta=0$ for each SNP. The Manhattan and quantile-quantile $(\mathrm{Q}-\mathrm{Q})$ plots were drawn with the "qqman" package in R. Moreover, correction for population stratification was evaluated by calculating the genomic inflation factor $\lambda$ with the "GenABEL" package [32].

We calculated genome-wide significance P-value thresholds with the simpleM method implemented in a R script for multiple testing correction [33]. simpleM calculation resulted in 72,648 independent effective tests and the genome-wide and suggestive significance values were then calculated as $6.88 \times 10^{-7}(0.05 / 72,648)$ and $1.38 \times 10^{-5}(1.00 / 72,648)$, respectively. The contribution of significant SNPs to the phenotypic variance was estimated by a restricted maximum likelihood (REML) method implemented in the GCTA v1.91 software [34]. Linkage disequilibrium (LD) analysis was performed for the significant SNPs using the solid spin algorithm implemented in Haploview version 4.2 [35].

\section{Identification of candidate genes}

Candidate genes for resistance to SP were identified based on functional annotation and expression level data. First, we performed functional annotation by searching for candidate genes within $500 \mathrm{~kb}$ regions on either side of the lead significant SNP with the help of the Variant Effect Predictor [36] and Biomart tools supported by Ensembl (http://www.ensembl.org) using the Gallus_gallus-5.0 genes dataset. Then, we investigated 
the biological functions of these candidate genes on PubMed (https://www.ncbi.nlm.nih.gov/pubmed).

\section{Gene expression analysis}

The gene expression levels in the spleen of infected and mock-infected birds (54 samples) were determined by RNA-Seq (three replicates) at three time points [4, 10 and 21 days post-infection (dpi)] for the three breeds. Spleen tissue was sampled and immediately preserved in RNAlater (Ambion) at room temperature for $24 \mathrm{~h}$ and then at $-20{ }^{\circ} \mathrm{C}$ until RNA extraction. Total RNA was extracted using the Trizol reagent (Invitrogen, Carlsbad, CA, USA) following the manufacturer's protocol. Libraries for each sample were prepared and sequenced on an Illumina HiSeq 2500 platform (Illumina Inc., San Diego, CA, USA), and 150 bp pairedend reads were generated. Raw reads were filtered and trimmed using fastp (version 0.19.1) [37]. Clean reads were mapped to the chicken reference genome (galGal5) using the HISAT2 program [38]. Reads mapped to a gene were counted with featureCounts [39] against the gene annotation from Ensembl database. The gene counts were normalized by the Bioconductor package DESeq2 [40] and differential expression P-values of candidate genes were then calculated with default parameters. To evaluate the correlation between time and gene expression levels of the control group, we conducted regression analysis by fitting the linear model with the function implemented in the R software version 3.5.1 (Foundation for Statistical Computing, Vienna, Austria).

\section{Genomic inbreeding analysis}

Inbreeding has long been reported to affect fitness traits such as resistance to disease [41-45]. To investigate the relationship between inbreeding and resistance to SP, we estimated the genomic inbreeding coefficients by measuring the genome-wide distribution of runs of homozygosity $(\mathrm{ROH})$ using the PLINK software. The genomic inbreeding coefficient $\mathrm{F}_{\mathrm{ROH}}$ was calculated as the proportion of the genome which is found in runs of homozygosity. The total physical length of all autosomes from the first to the last SNP is $930,016,100 \mathrm{bp}$. A ROH is defined as a segment of consecutive homozygous DNA that meets the following criteria: (1) a minimum size of $1000 \mathrm{~kb}$ and at least 50 homozygous SNPs; (2) a maximum of five missing SNPs allowed in a ROH; (3) a maximum of one heterozygous SNP per $\mathrm{ROH}$, so that $\mathrm{ROH}$ segments are not disrupted by an occasional heterozygous SNP; and (4) a maximum gap between SNPs of $1000 \mathrm{~kb}$ to ensure that SNP density does not affect a $\mathrm{ROH}$.
Table 1 Phenotype composition of the three chicken lines used in the GWAS

\begin{tabular}{lcccc}
\hline Breed & Died & Carrier state & Clearance & Total \\
\hline BY & 27 & 0 & 50 & 77 \\
DW & 89 & 77 & 198 & 364 \\
RIR & 84 & 84 & 209 & 377 \\
Total & 200 & 161 & 457 & 818 \\
\hline
\end{tabular}

$B Y$ Beijing You, DW Dwarf Chicken, RIR Rhode Island Red. These are the number of chicks that died, carried and cleared the pathogen, respectively

\section{Results}

Phenotype statistics and population structure

The details of the results of the SP challenge were previously reported [3]. Briefly, mortality rates for the RIR, BY, and DW chicks reached $25.1,8.3$, and $22.7 \%$, respectively, and the corresponding carrier-state levels in the spleens were equal to $17.9,0.6$, and $15.8 \%$, which indicate that BY chicks are more resistant to SP infection than DW and RIR chicks. Only part of the chicks that participated in the SP challenge test were genotyped. Since nearly all the BY chicks that survived could clear the pathogen (except three), we did not genotype the carriers in this breed. In the group of chicks that died, only the individuals that had conclusive symptoms and for which high quality DNA was available were genotyped. Furthermore, in the group of chicks that cleared the pathogen, we removed randomly some individuals to match the corresponding phenotype proportion. In total, 842 samples were genotyped and after a series of strict quality control procedures, 24 samples were eliminated because of a low genotyping call rate. Thus, the final GWAS population consisted of 818 samples (392 males and 426 females). The phenotypic composition of each line is given in Table 1. Based on the PCA plot (Fig. 1), three subpopulations are clearly distinguished, which indicates that population stratification can be accounted for in the linear

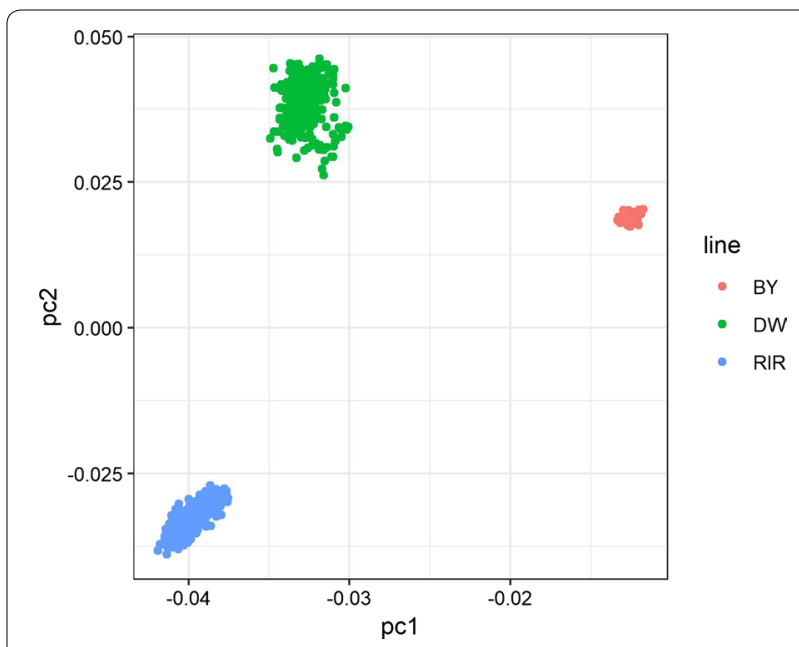

Fig. 1 PCA plot for population structure. The red, green and blue dots represent BY, DW and RIR individuals, respectively 
mixed model of the GWAS by including these principal components as covariates in the analysis.

\section{GWAS for the death trait}

The Manhattan and Q-Q plots for the death trait are in Fig. 2a. The single-marker analysis identified 42 SNPs that were significantly associated with death at the suggestive association threshold. Of these 42 SNPs, 17 genomewide significant SNPs spanned a narrow $0.55 \mathrm{Mb}$ region (33.48-34.03 Mb) on chicken chromosome GGA4 (GGA for Gallus gallus), which was the highest peak. Based on SNP annotation (Table 2), we found that 10 of the

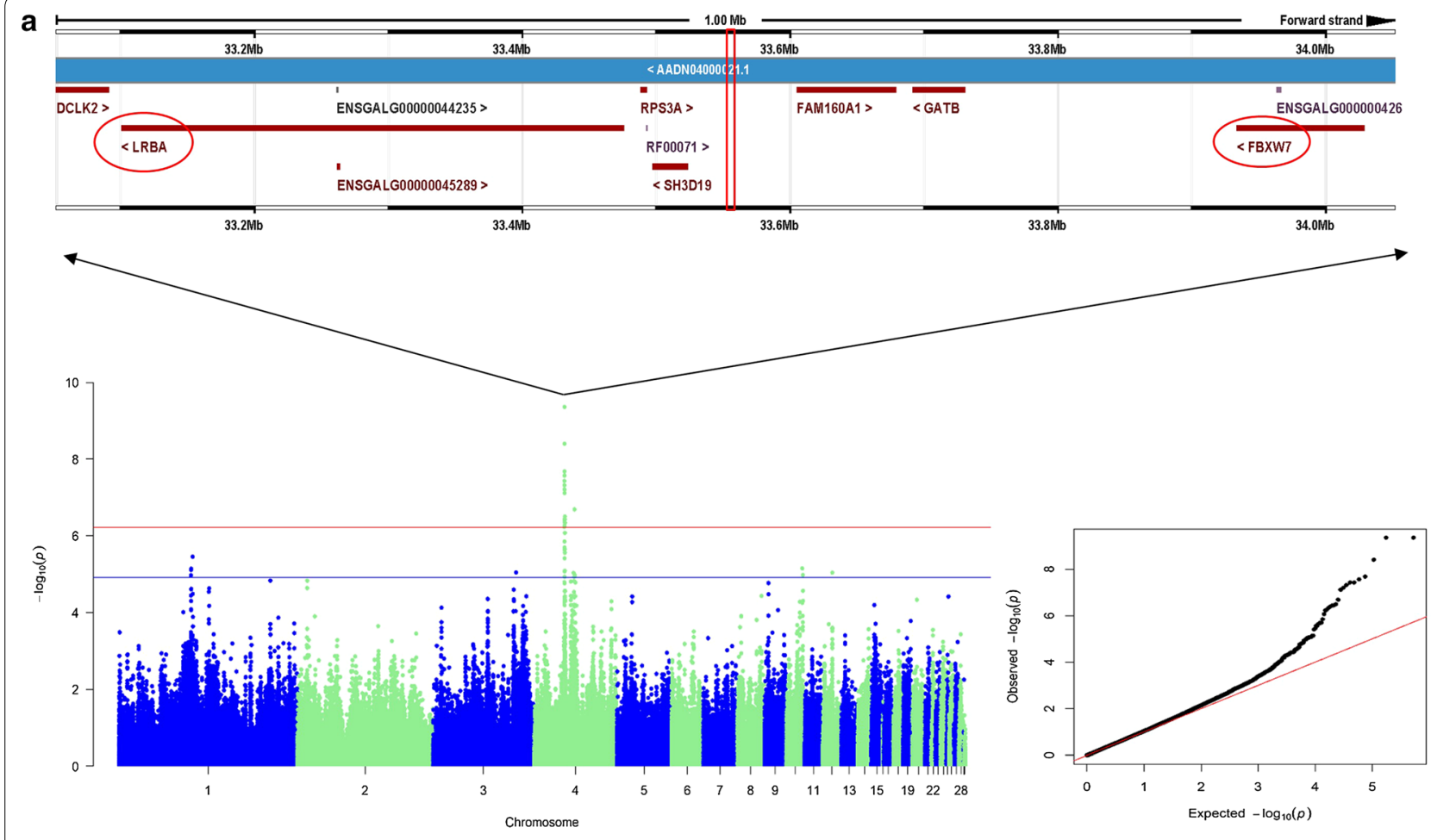

b

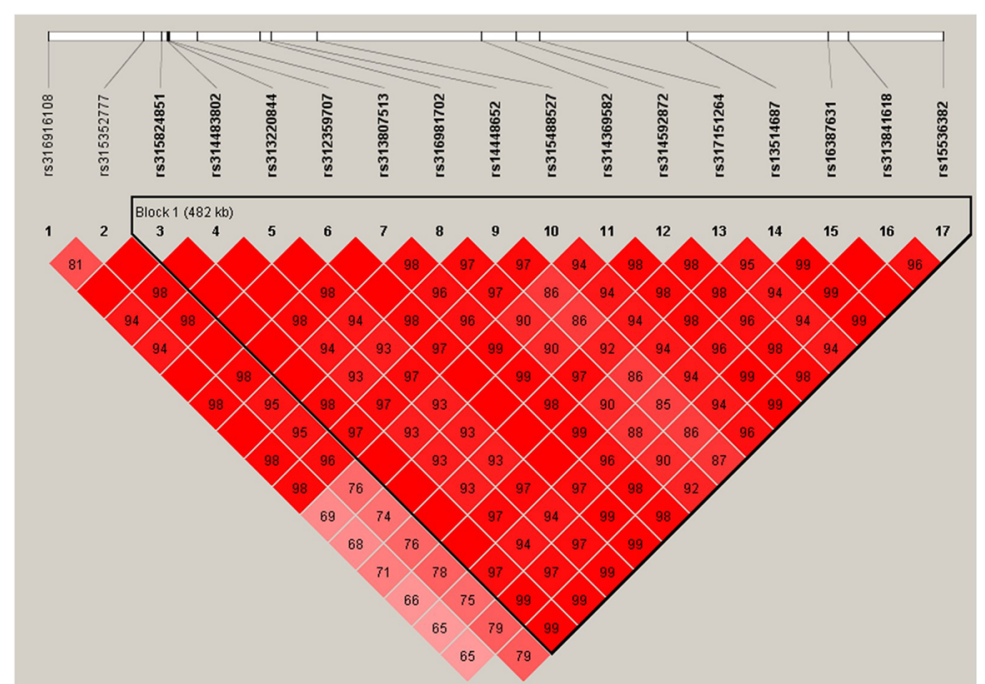

Fig. 2 GWAS results for death. a Manhattan plot and Q-Q plot. Each dot on this figure corresponds to a SNP within the dataset, while the horizontal red and blue lines denote the genome-wide significance $\left(6.88 \times 10^{-7}\right)$ and suggestive significance thresholds $\left(1.38 \times 10^{-5}\right)$, respectively. The Manhattan plot contains - log10 observed P-values for genome-wide SNPs (y-axis) plotted against their corresponding position on each chromosome (x-axis), while the Q-Q plot contains expected - log10-transformed P-values plotted against observed - log 10 -transformed P-values. b LD plots for significant SNPS 
Table 2 Genome-wide significant SNPs for death and suggestive significant SNPs for carrier-state

\begin{tabular}{|c|c|c|c|c|c|c|}
\hline Trait & SNP & GGA & Position & P-value & Annotation & Gene \\
\hline \multirow[t]{21}{*}{ Death } & rs316916108 & 4 & $33,478,824$ & $2.11 \mathrm{E}-08$ & Upstream & LRBA \\
\hline & rs315352777 & 4 & $33,538,092$ & $5.85 \mathrm{E}-07$ & intergenic & \\
\hline & rs315824851 & 4 & $33,548,952$ & $3.72 \mathrm{E}-08$ & Intergenic & \\
\hline & rs314483802 & 4 & $33,552,203$ & $4.38 \mathrm{E}-10$ & Intergenic & \\
\hline & rs313220844 & 4 & $33,553,077$ & $4.38 \mathrm{E}-10$ & Intergenic & \\
\hline & rs312359707 & 4 & $33,554,042$ & $3.72 \mathrm{E}-08$ & Intergenic & \\
\hline & rs313807513 & 4 & $33,571,056$ & $6.25 \mathrm{E}-08$ & Intergenic & \\
\hline & rs316981702 & 4 & $33,610,050$ & $3.96 \mathrm{E}-09$ & Intron & FAM160A1 \\
\hline & rs14448652 & 4 & $33,616,458$ & $2.73 \mathrm{E}-08$ & Intron & FAM $160 A 1$ \\
\hline & rs315488527 & 4 & $33,644,936$ & $4.80 \mathrm{E}-08$ & Intron & FAM $160 A 1$ \\
\hline & rs314369582 & 4 & $33,746,779$ & $5.43 \mathrm{E}-07$ & Intergenic & \\
\hline & rs314592872 & 4 & $33,768,246$ & $3.61 \mathrm{E}-07$ & Intergenic & \\
\hline & rs317151264 & 4 & $33,782,635$ & $4.02 \mathrm{E}-07$ & Intergenic & \\
\hline & rs13514687 & 4 & $33,873,598$ & 7.70E-08 & Intergenic & \\
\hline & rs16387631 & 4 & $33,960,862$ & 4.64E-07 & Intron & FBXW7 \\
\hline & rs313841618 & 4 & $33,973,296$ & $3.16 \mathrm{E}-07$ & Intron & FBXW7 \\
\hline & rs15536382 & 4 & $34,031,794$ & $3.73 \mathrm{E}-07$ & Upstream & FBXW7 \\
\hline & rs313296110 & 4 & $43,491,648$ & $2.53 \mathrm{E}-07$ & Intergenic & \\
\hline & rs317612144 & 4 & $43,491,692$ & $2.39 E-07$ & Intergenic & \\
\hline & rs318239967 & 4 & $43,491,742$ & $3.30 E-07$ & Intergenic & \\
\hline & rs313303913 & 4 & $44,551,373$ & $2.05 E-07$ & Upstream & GPM6A \\
\hline \multirow[t]{3}{*}{ Carrier-state } & rs312524326 & 5 & $50,147,152$ & $9.52 \mathrm{E}-06$ & Intergenic & \\
\hline & rs312970356 & 2 & $23,295,087$ & $9.65 \mathrm{E}-06$ & Intergenic & \\
\hline & rs317601331 & 5 & $49,848,247$ & $1.09 E-05$ & Intergenic & \\
\hline
\end{tabular}

significant SNPs were located at intergenic regions, five were in introns and two were upstream of the coding sequences. Through a REML analysis implemented in the GCTA software, we found that the most significant SNP, i.e. rs314483802, $\left(\mathrm{P}=4.38 \times 10^{-10}\right.$; intergenic $)$ explained $11.73 \%$ of the phenotypic variance. An LD analysis revealed that all the genome-wide significant SNPs were in high LD (Fig. 2b), which makes it difficult to identify causal SNPs. The genomic control inflation factor $(\lambda)$ calculated for the death trait was equal to 1.08 , which is a little higher than the ideal value of 1 and indicates a mild but acceptable population stratification. We identified three genes that involved the eight upstream and intronic SNPs: family with a sequence similarity 160 member A1 (FAM160A1), F-box and WD repeat domain containing 7 (FBXW7), and LPS responsive beige-like anchor protein (LRBA). FAM10A1 is a protein coding gene about which little is known in the literature. $F B X W 7$ modulates the $\mathrm{NF}-\mathrm{KB}$ signaling pathway by targeting $N F-\kappa B 2$ for ubiquitination and destruction $[46,47]$. NF- $\mathrm{kB}$ is one of the most important signaling pathways of the inflammation and immune system. According to Fukushima et al. [47], the depletion of $F b w 7$ (synonymous to $F B X W 7$ ) in mice leads to reduced NF- $\mathrm{KB}$ activity and perturbed $\mathrm{T}$ cell differentiation. Thus, $F B X W 7$ is a very promising candidate gene that may affect immune response after SP infection. $L R B A$ is an important gene that is involved in a syndrome of immune deficiency and autoimmunity. Deleterious mutations in $L R B A$ cause defects in B cell activation and autophagy, and can increase susceptibility to apoptosis [48]. Furthermore, a new study has recently linked $L R B A$ to the NF-kB immune pathway [49].

Our gene expression data provided further evidence that supports implication of these candidate genes during a SP infection. As shown in Fig. 3, expression of FBXW7 was significantly downregulated after SP infection at all three time points in RIR chicks. Although this downregulation was not significant in BY chicks and was significant at $21 \mathrm{dpi}$ in DW chicks, overall the same trend was observed in the three lines. Expression of $L R B A$ was downregulated at a later time post-infection in all three lines. Interestingly, these two genes showed a timedependent expression in the control group. To confirm this, we performed a regression analysis of gene expression levels and time (see Fig. 4) that showed that the expression levels of $L R B A$ and $F B X W 7$ are positively correlated with time, which is consistent with the fact that the chicks are considerably more resistant to SP when 


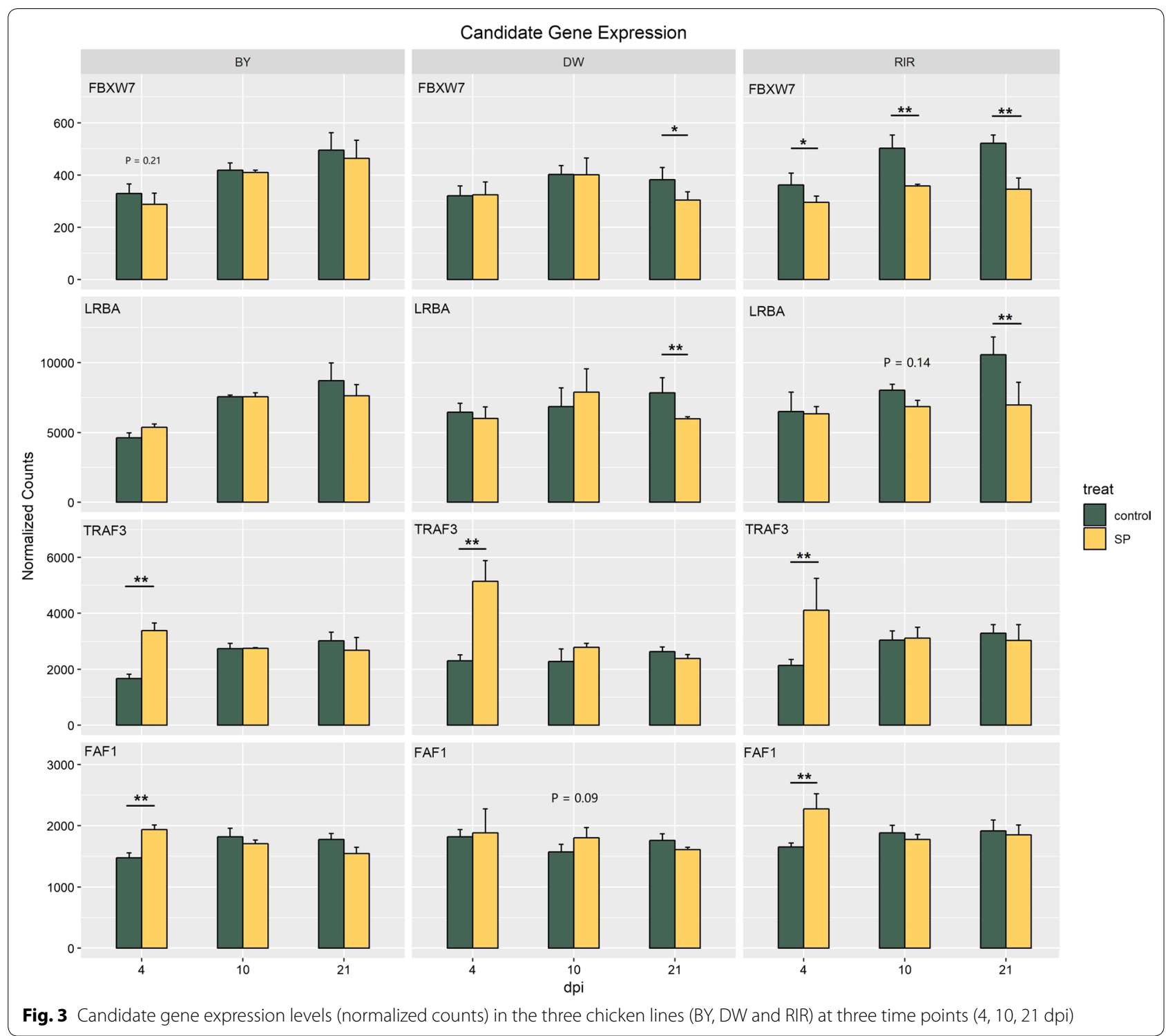

they are more than 20 days old. Our results provide more evidence that FBXW7 and $L R B A$ are associated with resistance to SP.

\section{GWAS for the carrier state}

In the GWAS for the carrier-state trait, the linear mixed model GWAS could not identify genome-wide significant SNPs. However, three SNPs were above the level of suggestive significance (Fig. 5). The genomic control inflation factor $(\lambda)$ for carrier-state was equal to 1.07, which is similar to the value found for death. Two of these three potential SNPs are located on GGA5 and the other one on GGA2. The rs312524326 SNP on GGA5 was located close to several candidate genes including the TNF receptor associated factor 3 gene (TRAF3).
TRAF3 participates in the signal transduction of CD40, a member of the TNFR family that is important for the activation of immune response [50]. Besides, TRAF3 controls the activation of the canonical and alternative NF- $\mathrm{B}$ signaling pathway via the lymphotoxin beta receptor [51]. In our experiments, the expression level of TRAF3 was up-regulated at $4 \mathrm{dpi}$ in all three lines; however, it showed no difference at later times (Fig. 3). The SNP on GGA2 (rs312970356) was close to the miRNA gga-mir-489. Expression of gga-mir-489 increases in CD30hi cells and is connected to Marek's disease herpesvirus infection [52]. We used the miRDB tool [53, 54] to predict the target genes for gga-mir-489 and found that one of the predicted target genes was FAS associated factor 1 (FAF1) with a high target score of 98. The protein encoded by FAF1 binds to the FAS antigen (TNFRSF6) 

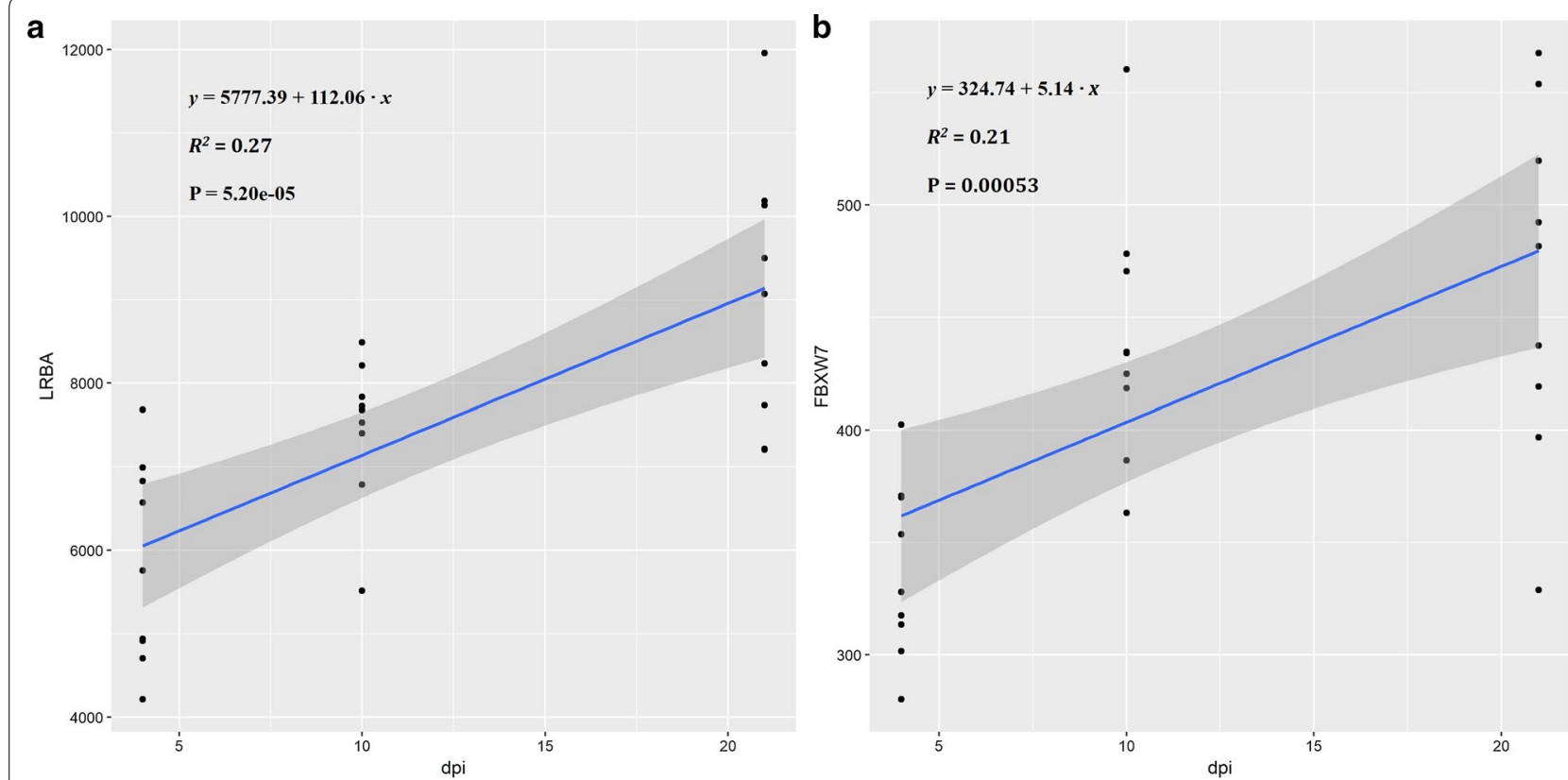

Fig. 4 Regression analysis between gene normalized counts and time. The analysis was conducted using a linear model for a LRBA, lipopolysacchari de-responsive and beige-like anchor protein and $\mathbf{b}$ FBXW7, F-box and WD repeat domain containing 7

and can initiate apoptosis or enhance apoptosis via the FAS antigen. Down-regulation of FAF1 can activate the TNF- $\alpha / N F-\kappa B$ signaling pathway [55]. We found that the expression of $F A F 1$ was up-regulated at 4 dpi in BY and RIR chicks after SP infection in our study (Fig. 3).

\section{Runs of homozygosity}

We found that $F_{\mathrm{ROH}}$ did not differ between the traits analyzed here (Fig. 6), but the DW and BY chicks that died of SP infection showed the lowest values. In contrast, at the breed level, all comparisons of genomic inbreeding coefficient involving the RIR breed were significant.

\section{Discussion}

Salmonella infections are a serious health risk to both humans and animals. In humans, typhoid fever outbreaks occur every few years and cause significant morbidity and mortality [56]. A human GWAS for Salmonella resistance conducted between infected individuals and a large control population reported a significant association with the MHC region [57]. Non-human research has the potential advantage of obtaining accurate phenotypes through bacterial challenge tests. However, only a few successful studies have been reported on infectious diseases owing to the difficulty in phenotyping the affected individuals and the complex genetic architecture of diseases. With the development of animal genomics, more domestic animals are being used as models for biomedical research [58].

To the best of our knowledge, this is one of the few large-scale GWAS, which have been carried out on both the death and carrier-state traits following a well-organized SP challenge experiment. We investigated the genetics of resistance to SP in 818 pure-bred chicks from three chicken lines by genotyping 302,927 SNPs from a highdensity chip and performing a GWAS. Although difficult, it would be highly useful to find a general mechanism of disease resistance among populations; towards this aim, we used three chicken lines with different genetic backgrounds. We identified a strong association at a region that was located between 33.48 and $34.03 \mathrm{Mb}$ on GGA4 for death, and two suggestive signals on GGA5 and GGA2 for carrier state. Combining the biological functions of the genes found in these regions and RNAseq data, we identified four candidate genes that could account for resistance to SP, namely $F B X W 7$ and $L R B A$ for death, and TRAF3 and gga-mir-489 (targeting FAF1) for carrier state. Interestingly, these four genes have been reported to participate in the NF- $\mathrm{KB}$ signaling pathway $[47,49,51,55]$, which suggests that this pathway may play a central role in the host's resistance to SP infection. As a major transcription factor, NF-kB regulates many genes that are involved in both the innate and adaptive immune response [59]. Because SP is an intracellular bacterium, we suspect that the $\mathrm{T}$ cell development process related to 


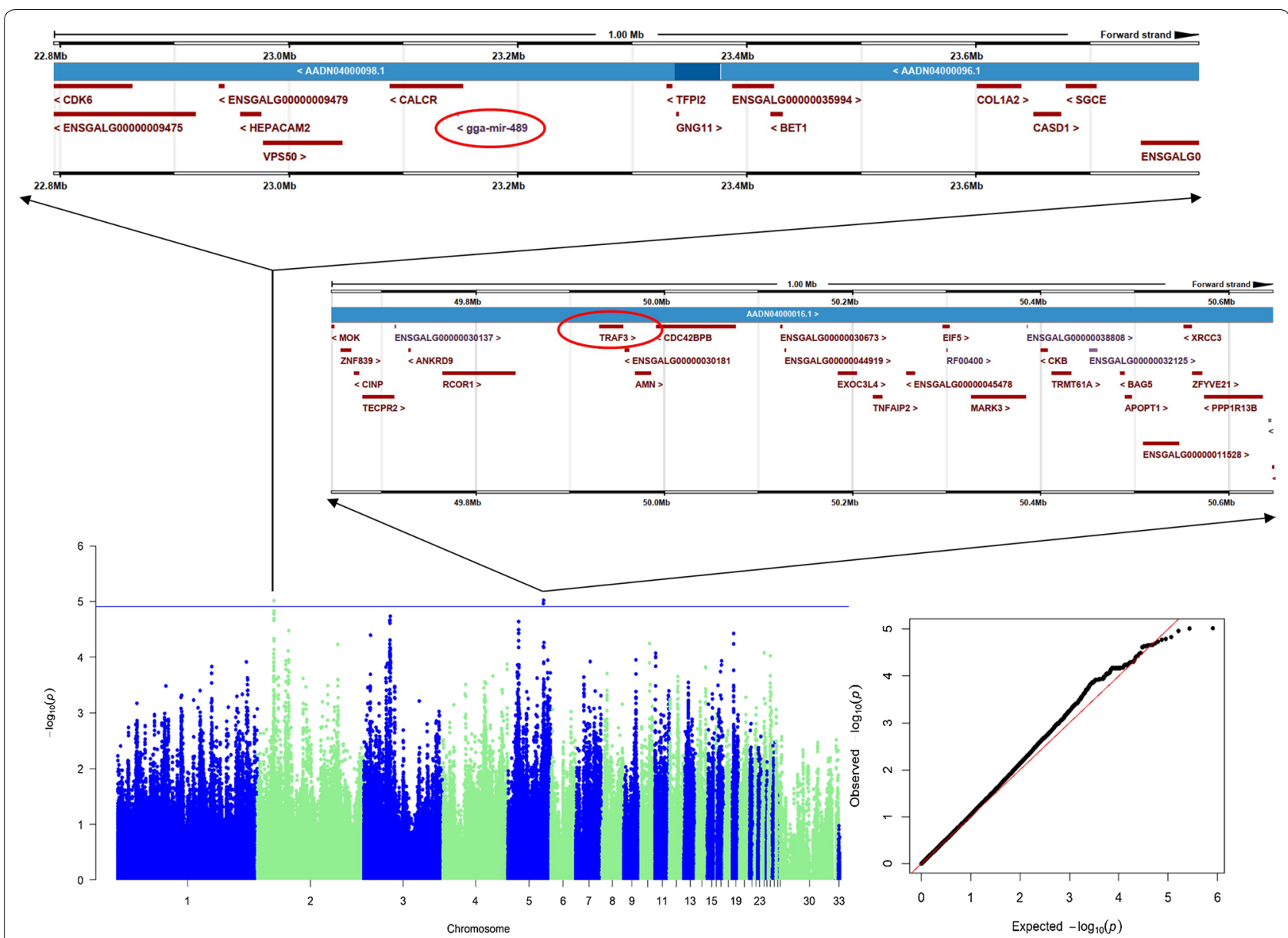

Fig. 5 Manhattan plot and Q-Q plot for carrier-state. Each dot on this figure corresponds to a SNP within the dataset, while the horizontal blue line denotes the suggestive significance thresholds $\left(1.38 \times 10^{-5}\right)$

the NF- $\mathrm{kB}$ signaling pathway may constitute the underlying mechanism of resistance to SP and thus deserves more research.

Until now, many Salmonella resistance genes have been identified in fowl [19], for example, TLR4 and SLC11A1. However, most of these studies were carried out within a single population. A GWAS performed across multiple populations can increase the power to detect novel loci and achieve a higher mapping resolution than in previous studies [60-62]. The regions and gene candidates reported in our study have not been detected in previous studies on resistance to Salmonella infection, which may be explained by the fact that we used different chicken lines and Salmonella strains. The immunobiology of typhoidal and non-typhoidal Salmonella diseases differs substantially [63], and these diseases correspond to different host genetic resistances; thus, it is important to compare the mechanisms for different Salmonella diseases. Our study provided new data for the genetic determinism of typhoidal Salmonella resistance. Because disease resistance is a complex trait, it is likely that there are many more disease-related genes that could not be identified here due to the limited sample size.

The candidate genes identified in our study showed a time- and breed-related expression, which might be linked to different development stages of the affected individual. Analysis of the expression data reveals the complexity of the transcription of these genes at the different time points and in the different breeds. In the SP challenge test, the BY chicks were the most resistant to SP; however, RIR chicks (the most susceptible breed) showed more consistent results at different time points. Interestingly, the association between the candidate genes and resistance to SP was stronger in the RIR chicks with high susceptibility.

BY is a local breed of chickens with the highest level of resistance to the disease and the lowest inbreeding coefficient; DW is a synthetic line with both a low level of resistance to and a low inbreeding coefficient; RIR is the most intensively-selected line with a low level of 


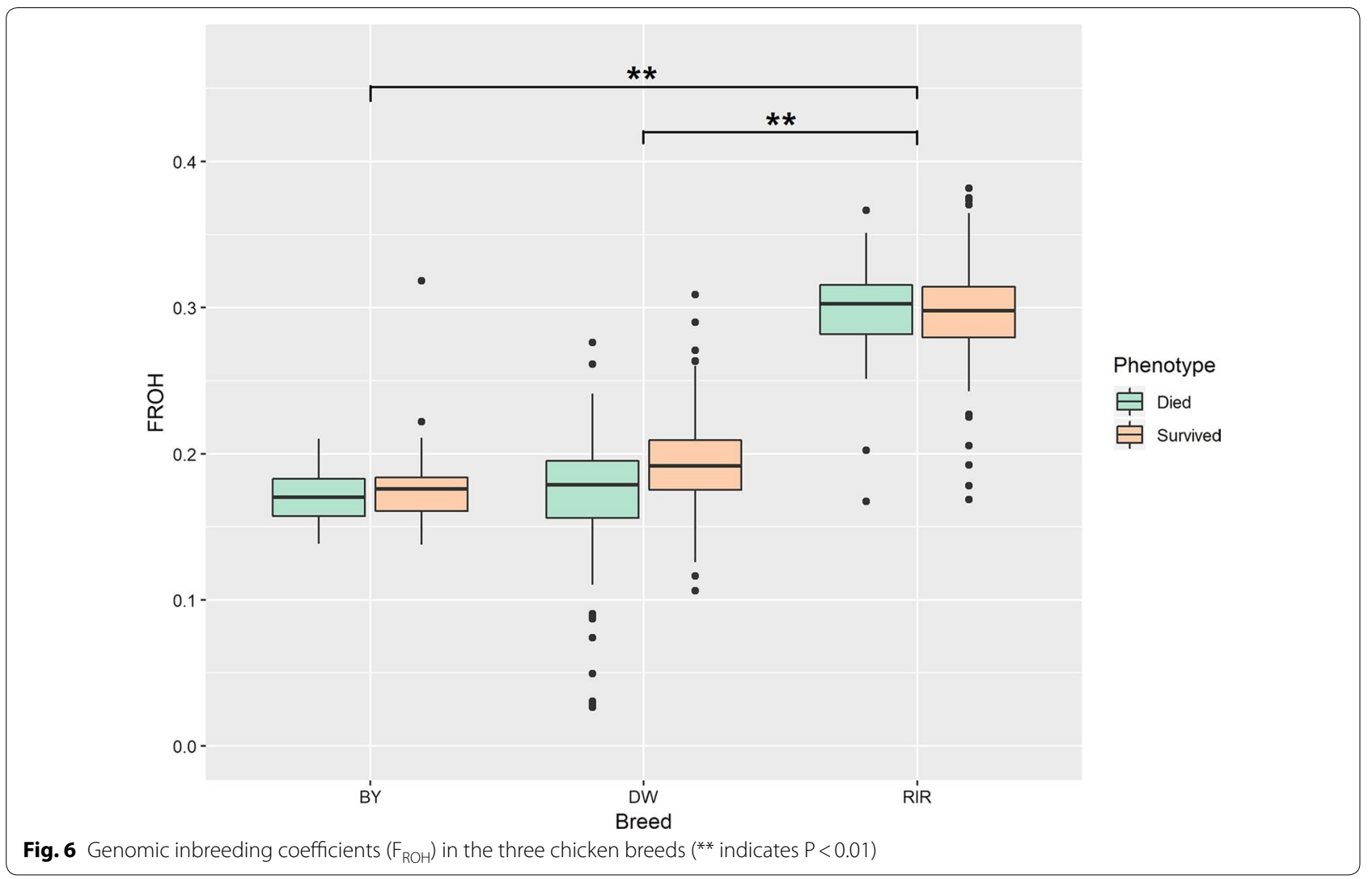

resistance to SP and a high inbreeding coefficient. Many studies have reported that inbreeding is associated with disease resistance $[43,64-66]$, which is not the case in our study. One of the reasons could be that inbreeding depression in farm animals is more complex and different from that in wild populations.

Considerably more research is required before we can completely understand the genetics of any complex trait, especially traits that concern elaborate immunological functions. In this study, we show the feasibility of using a domestic animal for mapping the genomic regions that underlie an infectious disease and for providing new information that will be useful in subsequent comparative immunology studies. The genetic determinism of immunity is extremely complex and shaped by the contribution of multiple genes and environmental factors [67]. Therefore, more GWAS on domestic animals will help identify genes that are involved in the immunity mechanisms occurring during infectious diseases.

\section{Conclusions}

Our GWAS on death and carrier state after SP infection identified new loci and genes associated with resistance to SP. The NF- $\mathrm{kB}$ signaling pathway is likely to play a central role in immunity against SP. These results provide new information on the genetic determinism of the resistance to infectious diseases; offer theoretical basis for breeding SP-resistant chicks using marker-assisted selection; and provide new data for research on salmonellosis in humans and other animals.

\section{Abbreviations}

BY: Beijing You; DW: Dwarf Chicken; FAF1: FAS associated factor 1; FBXW7: F-box and WD repeat domain containing 7; GWAS: genome-wide association study; LD: linkage disequilibrium; LMM: linear mixed model; LRBA: lipopolysaccharide-responsive and beige-like anchor protein; NCBI: National Center for Biotechnology Information; NF-kB: nuclear factor kappa-light-chain-enhancer of activated B cells; PCA: principal component analysis; PCR: polymerase chain reaction; RIR: Rhode Island Red; RNA-Seq: RNA sequencing; SNP: single-nucleotide polymorphism; SP: Salmonella pullorum; TRAF3: TNF receptor associated factor 3 .

\section{Acknowledgements}

This work was supported by the earmarked fund for the Beijing Innovation Team of the Modern Agro-industry Technology Research System (BAIC042018, BAIC04-2019), National Natural Science Foundation of China (31772581) and Chinese Agricultural Research System (CARS-41). We also gratefully acknowledge our colleagues in the Poultry Team at the National Engineering Laboratory for Animal Breeding of China Agricultural University, for their assistance on sample collection and helpful comments on the study, and 
Pedro Almeida from University College London, for his kind help to polish the writing of the manuscript.

\section{Authors' contributions}

$L Q, Z N$ and $L B$ conceived and designed the research. XHL performed the experiments, analyzed and interpreted the data, and wrote the manuscript. $\mathrm{CN}, \mathrm{YJ}$ and $\mathrm{YL}$ performed the experiments and interpreted the data. $\mathrm{YC}, \mathrm{CZ}$ $X Z L, L W$, JZ and KL interpreted the data and contributed reagents and materials. All authors read and approved the final manuscript.

\section{Data availability}

The data on the 54 chickens used in RNA-Seq analysis are accessible at the National Center for Biotechnology Information (NCBI) under BioProject accession number PRJNA511038.

\section{Ethics approval and consent to participate}

All experiments were approved by the Committee for Animal Care and Use of China Agricultural University (Approval ID: XXCB-20090209). All the animals were fed and handled according to the regulations and guidelines established by this committee, and all efforts were made to minimize suffering.

\section{Competing interests}

The authors declare that they have no competing interests.

\begin{abstract}
Author details
${ }^{1}$ State Key Laboratory of Animal Nutrition, Department of Animal Genetics and Breeding, National Engineering Laboratory for Animal Breeding, College of Animal Science and Technology, China Agricultural University, Beijing, China. ${ }^{2}$ Beijing Municipal General Station of Animal Science, Beijing, China. ${ }^{3}$ Institute of Animal Sciences, Chinese Academy of Agricultural Sciences, Beijing, China. ${ }^{4}$ Department of Grassland Science, China Agricultural University, Beijing, China.
\end{abstract}

Received: 27 February 2019 Accepted: 29 August 2019

Published online: 18 September 2019

\section{References}

1. Hohmann EL. Nontyphoidal salmonellosis. Clin Infect Dis. 2001;32:263-9.

2. Barrow PA, Freitas Neto OC. Pullorum disease and fowl typhoid-new thoughts on old diseases: a review. Avian Pathol. 2011;40:1-13.

3. Li X, Nie C, Zhang Z, Wang Q, Shao P, Zhao Q, et al. Evaluation of genetic resistance to Salmonella pullorum in three chicken lines. Poult Sci. 2018;97:764-9.

4. Chappell L, Kaiser P, Barrow P, Jones MA, Johnston C, Wigley P. The immunobiology of avian systemic salmonellosis. Vet Immunol Immunopathol. 2009;128:53-9.

5. Wigley P, Berchieri A, Page KL, Smith AL, Barrow PA. Salmonella enterica Serovar Pullorum persists in splenic macrophages and in the reproductive tract during persistent, disease-free carriage in chickens. Infect Immun. 2001;69:7873-9.

6. Pan Z, Wang X, Zhang X, Geng S, Chen X, Pan W, et al. Changes in antimicrobial resistance among Salmonella enterica subspecies enterica serovar Pullorum isolates in China from 1962 to 2007. Vet Microbiol. 2009;136:387-92.

7. Cheng HH, Kaiser P, Lamont SJ. Integrated genomic approaches to enhance genetic resistance in chickens. Annu Rev Anim Biosci. 2013;1:239-60.

8. Hutt FB, Scholes JC. Genetics of the fowl XIII. Breed differences in susceptibility to Salmonella pullorum. Poult Sci. 1941;20:342-52.

9. Bumstead N, Barrow PA. Genetics of resistance to Salmonella typhimurium in newly hatched chicks. Br Poult Sci. 1988;29:521-9.

10. Bumstead N, Barrow P. Resistance to Salmonella gallinarum, S. pullorum, and S. enteritidis in inbred lines of chickens. Avian Dis. 1993;37:189-93.

11. Protais J, Colin P, Beaumont C, Guillot JF, Lantier F, Pardon P, et al. Line differences in resistance to Salmonella enteritidis PT4 infection. Br Poult Sci. 1996;37:329-39.

12. Berthelot F, Beaumont C, Mompart F, Girard-Santosuosso O, Pardon $P$, Duchet-Suchaux M. Estimated heritability of the resistance to cecal carrier state of Salmonella enteritidis in chickens. Poult Sci. 1998;77:797-801.
13. Beaumont C, Protais J, Guillot JF, Colin P, Proux K, Millet N, et al. Genetic resistance to mortality of day-old chicks and carrier-state of hens after inoculation with Salmonella enteritidis. Avian Pathol. 1999;28:131-5.

14. Zekarias B, Ter Huurne AA, Landman WJM, Rebel JMJ, Pol JMA, Gruys E. Immunological basis of differences in disease resistance in the chicken. Vet Res. 2002;33:109-25.

15. Li DF, Lian L, Qu LJ, Chen YM, Liu WB, Chen SR, et al. A genome-wide SNP scan reveals two loci associated with the chicken resistance to Marek's disease. Anim Genet. 2013:44:217-22.

16. Wolc A, Arango J, Jankowski T, Settar P, Fulton JE, O'Sullivan NP, et al. Genome-wide association study for Marek's disease mortality in layer chickens. Avian Dis. 2013;57:395-400.

17. Zhang L, Li P, Liu R, Zheng M, Sun Y, Wu D, et al. The identification of loci for immune traits in chickens using a genome-wide association study. PLOS ONE. 2015;10:e0117269.

18. Psifidi A, Banos G, Matika O, Desta TT, Bettridge J, Hume DA, et al. Genome-wide association studies of immune, disease and production traits in indigenous chicken ecotypes. Genet Sel Evol. 2016;48:74.

19. Calenge F, Kaiser P, Vignal A, Beaumont C. Genetic control of resistance to salmonellosis and to Salmonella carrier-state in fowl: a review. Genet Sel Evol. 2010;42:11.

20. Kranis A, Gheyas AA, Boschiero C, Turner F, Yu L, Smith S, et al. Development of a high-density $600 \mathrm{~K}$ SNP genotyping array for chicken. BMC Genomics. 2013;14:59.

21. Li Q, Hu Y, Xu Y, Chen J, Fang L, Liu Z, et al. A gene knock-in method used to purify plasmid pSPI12 from Salmonella enterica serovar Pullorum and characterization of IpaJ. J Microbiol Methods. 2014;98:128-33.

22. Purcell S, Neale B, Todd-Brown K, Thomas L, Ferreira MAR, Bender D, et al. PLINK: a tool set for whole-genome association and population-based linkage analyses. Am J Hum Genet. 2007;81:559-75.

23. Browning BL, Browning SR. A unified approach to genotype imputation and haplotype-phase inference for large data sets of trios and unrelated individuals. Am J Hum Genet. 2009;84:210-23.

24. Astle W, Balding DJ. Population structure and cryptic relatedness in genetic association studies. Stat Sci. 2009;24:451-71.

25. Yu J, Pressoir G, Briggs WH, Vroh Bi I, Yamasaki M, Doebley JF, et al. A unified mixed-model method for association mapping that accounts for multiple levels of relatedness. Nat Genet. 2006;38:203-8.

26. Kang HM, Zaitlen NA, Wade CM, Kirby A, Heckerman D, Daly MJ, et al. Efficient control of population structure in model organism association mapping. Genetics. 2008;178:1709-23.

27. Kang HM, Sul JH, Service SK, Zaitlen NA, Kong S, Freimer NB, et al. Variance component model to account for sample structure in genomewide association studies. Nat Genet. 2010;42:348-54.

28. Zhang Z, Ersoz E, Lai C-Q, Todhunter RJ, Tiwari HK, Gore MA, et al. Mixed linear model approach adapted for genome-wide association studies. Nat Genet. 2010;42:355-60.

29. Lippert C, Listgarten J, Liu Y, Kadie CM, Davidson RI, Heckerman D. FaST linear mixed models for genome-wide association studies. Nat Methods. 2011;8:833-5.

30. Zhou X, Stephens M. Genome-wide efficient mixed-model analysis for association studies. Nat Genet. 2012;44:821-4.

31. Loh PR, Tucker G, Bulik-Sullivan BK, Vilhjálmsson BJ, Finucane HK, Salem RM, et al. Efficient Bayesian mixed-model analysis increases association power in large cohorts. Nat Genet. 2015;47:284-90.

32. Aulchenko YS, Ripke S, Isaacs A, van Duijn CM. GenABEL: an R library for genome-wide association analysis. Bioinformatics. 2007;23:1294-6.

33. Gao X, Becker LC, Becker DM, Starmer JD, Province MA. Avoiding the high Bonferroni penalty in genome-wide association studies. Genet Epidemiol. 2010;34:100-5.

34. Yang J, Lee SH, Goddard ME, Visscher PM. GCTA: a tool for genomewide complex trait analysis. Am J Hum Genet. 2011;88:76-82.

35. Barrett JC, Fry B, Maller J, Daly MJ. Haploview: analysis and visualization of LD and haplotype maps. Bioinformatics. 2005;21:263-5.

36. McLaren W, Pritchard B, Rios D, Chen Y, Flicek P, Cunningham F. Deriving the consequences of genomic variants with the Ensembl API and SNP Effect Predictor. Bioinformatics. 2010;26:2069-70.

37. Chen S, Zhou Y, Chen Y, Gu J. fastp: an ultra-fast all-in-one FASTQ preprocessor. Bioinformatics. 2018;34:1884-90.

38. Kim D, Langmead B, Salzberg SL. HISAT: a fast spliced aligner with low memory requirements. Nat Methods. 2015;12:357-60. 
39. Liao Y, Smyth GK, Shi W. featureCounts: an efficient general purpose program for assigning sequence reads to genomic features. Bioinformatics. 2014;30:923-30.

40. Love MI, Huber W, Anders S. Moderated estimation of fold change and dispersion for RNA-seq data with DESeq2. Genome Biol. 2014;15:550.

41. Stevens L, Yan G, Pray LA. Consequences of inbreeding on invertebrate host susceptibility to parasitic infection. Evolution. 1997;51:2032-9.

42. Acevedo-Whitehouse K, Gulland F, Greig D, Amos W. Inbreeding: disease susceptibility in California sea lions. Nature. 2003:422:35.

43. Calleri DV 2nd, McGrail Reid E, Rosengaus RB, Vargo EL, Traniello JFA. Inbreeding and disease resistance in a social insect: effects of heterozygosity on immunocompetence in the termite Zootermopsis angusticollis Proc Biol Sci. 2006;273:2633-40.

44. Armstrong DP, Cassey P. Estimating the effect of inbreeding on survival. Anim Conserv. 2007;10:487-92.

45. Charlesworth D, Willis JH. The genetics of inbreeding depression. Nat Rev Genet. 2009;10:783-96.

46. Arabi A, Ullah K, Branca RMM, Johansson J, Bandarra D, Haneklaus M, et al. Proteomic screen reveals Fbw7 as a modulator of the NF-KB pathway. Nat Commun. 2012:3:976

47. Fukushima H, Matsumoto A, Inuzuka H, Zhai B, Lau AW, Wan L, et al. SCF(Fbw7) modulates the NFkB signaling pathway by targeting NFkB2 for ubiquitination and destruction. Cell Rep. 2012;1:434-43.

48. Lopez-Herrera G, Tampella G, Pan-Hammarström Q, Herholz P, TrujilloVargas CM, Phadwal K, et al. Deleterious mutations in LRBA are associated with a syndrome of immune deficiency and autoimmunity. Am J Hum Genet. 2012;90:986-1001.

49. Wang JW, Reiser MA, Li K, Rifkin E, Wang B, Kolliputi N, et al. LRBA causes immunodeficiency and autoimmunity by deregulating NFkB-mediated multiple immune effectors critical for B cell activation. J Allergy Clin Immunol. 2014;133:AB251.

50. Ni CZ, Welsh K, Leo E, Chiou C, Wu H, Reed JC, et al. Molecular basis for CD40 signaling mediated by TRAF3. Proc Natl Acad Sci USA. 2000;97:10395-9.

51. Bista P, Zeng W, Ryan S, Bailly V, Browning JL, Lukashev ME. TRAF3 controls activation of the canonical and alternative NFKB by the lymphotoxin beta receptor. J Biol Chem. 2010;285:12971-8.

52. Kumar S, Kunec D, Buza JJ, Chiang HI, Zhou H, Subramaniam S, et al. Nuclear factor kappa B is central to Marek's disease herpesvirus induced neoplastic transformation of CD30 expressing lymphocytes in-vivo. BMC Syst Biol. 2012;6:123.

53. Wang $X$, El Naqa IM. Prediction of both conserved and nonconserved microRNA targets in animals. Bioinformatics. 2008;24:325-32.
54. Wang $X$. miRDB: a microRNA target prediction and functional annotation database with a wiki interface. RNA. 2008;14:1012-7.

55. Altomare DA, Menges CW, Pei J, Zhang L, Skele-Stump KL, Carbone M, et al. Activated TNF-a/NF-KB signaling via down-regulation of Fas-associated factor 1 in asbestos-induced mesotheliomas from Arf knockout mice. Proc Natl Acad Sci USA. 2009;106:3420-5.

56. Crump JA, Mintz ED. Global trends in typhoid and paratyphoid fever. Clin Infect Dis. 2010;50:241-6.

57. Dunstan SJ, Hue NT, Han B, Li Z, Tram TTB, Sim KS, et al. Variation at HLA-DRB1 is associated with resistance to enteric fever. Nat Genet. 2014;46:1333-6.

58. Andersson L. Domestic animals as models for biomedical research. Upsala J Med Sci. 2016;121:1-11.

59. Zhang Q, Lenardo MJ, Baltimore D. 30 years of NF-kB: a blossoming of relevance to human pathobiology. Cell. 2017;168:37-57.

60. van de Bunt M, Cortes A, IGAS Consortium, Brown MA, Morris AP, McCarthy MI. Evaluating the performance of fine-mapping strategies at common variant GWAS loci. PLoS Genet. 2015;11:e1005535.

61. Asimit JL, Hatzikotoulas K, McCarthy M, Morris AP, Zeggini E. Transethnic study design approaches for fine-mapping. Eur J Hum Genet. 2016;24:1330-6.

62. Tehranchi A, Hie B, Dacre M, Kaplow I, Pettie K, Combs P, et al. Finemapping cis-regulatory variants in diverse human populations. eLife. 2019;8:e39595.

63. Gal-Mor O, Boyle EC, Grassl GA. Same species, different diseases: how and why typhoidal and non-typhoidal Salmonella enterica serovars differ. Front Microbiol. 2014;5:391.

64. Onzima RB, Upadhyay MR, Doekes HP, Brito LF, Bosse M, Kanis E, et al. Genome-wide characterization of selection signatures and runs of homozygosity in Ugandan goat breeds. Front Genet. 2018;9:318.

65. Huisman J, Kruuk LEB, Ellis PA, Clutton-Brock T, Pemberton JM. Inbreeding depression across the lifespan in a wild mammal population. Proc Natl Acad Sci USA. 2016;113:3585-90.

66. Bérénos C, Ellis PA, Pilkington JG, Pemberton JM. Genomic analysis reveals depression due to both individual and maternal inbreeding in a freeliving mammal population. Mol Ecol. 2016;25:3152-68.

67. Lazzaro BP, Schneider DS. The genetics of immunity. Genetics. 2014;197:467-70.

\section{Publisher's Note}

Springer Nature remains neutral with regard to jurisdictional claims in published maps and institutional affiliations.
Ready to submit your research? Choose BMC and benefit from:

- fast, convenient online submission

- thorough peer review by experienced researchers in your field

- rapid publication on acceptance

- support for research data, including large and complex data types

- gold Open Access which fosters wider collaboration and increased citations

- maximum visibility for your research: over $100 \mathrm{M}$ website views per year

At BMC, research is always in progress.

Learn more biomedcentral.com/submissions 